\title{
Endoscopically assisted reduction of nasal bone fractures*
}

\author{
Chan Hum Park, Tec Keun Kwon, Jun Ho Lee, Sung Ju Hong, Ho Hoon Joung, \\ Hyung Seob Kim
}

Department of Otorhinolaryngology-Head and Neck Surgery, Facial Skeleton Bio Institute, School of Medicine, Hallym University, Chuncheon, Korea

\begin{abstract}
SUMMARY
The standard treatment for a nasal bone fracture is closed reduction within 10 days. After that time, callus and fibrous connective tissue will limit a precise reduction. This study evaluated endoscopically assisted reduction for the treatment of nasal bone fractures in patients who miss the optimal operating time. Fifteen patients underwent endoscopically assisted correction of nasal bone fractures. The surgery was performed with the patients under general anesthesia. An intercartilaginous incision was made. The depressed bony fragments were repositioned under endoscopic visualization. In all cases, good anatomic reduction was obtained, the postoperative course was uneventful, with no complications, and the patients were satisfied with the shape of their noses. Endoscopy appears to be the best tool for visualizing intraoperative repositioning control, enabling the surgeon to confirm a fracture site with callus and to perform an accurate reduction. Endoscopically assisted reduction provides an alternative option in the treatment of patients outside the optimal temporal window for surgery.
\end{abstract}

Key words: endoscopically assisted surgery, nasal bone fracture

\section{INTRODUCTION}

As the nose is the central, anterior-most projection of the face and the nasal bone is a small thin bone ${ }^{(1,2)}$ the vulnerability to trauma is great. Nasal fractures are thus the most common fracture of the facial region.

Although nasal fractures are managed in different ways, depending on the surgeon's preference and hospital protocols, the majority of nasal fractures are managed using closed reduction because it is generally safe and easy to perform. Many authors recommend performing the reduction within 10 days of the trauma because the timing of surgery may influence the final results ${ }^{(3)}$ but some patients do not seek reduction at this optimal time. Although closed reduction is very simple and an easy treatment method for nasal bone fractures, the reduction does not always restore the shape of the nose before the injury and patients may be dissatisfied with the shape of their nose. In those patients who do not achieve satisfactory results, rhinoplasty can be performed at a later date ${ }^{(4)}$. Many authors prefer to wait at least 6 months following the injury before proceeding, when some degree of stability at the fracture site has been achieved ${ }^{(3)}$. In other words, a delay occurs before the patient undergoes the appropriate treatment. Since the bone healing process and fibrotic change become factors limiting accurate reduction, the surgeon must have a precise diagnosis of the fracture to allow accurate reduction when treating patients undergoing delayed surgery.

This study evaluated whether endoscopically assisted reduction is useful for the treatment of nasal bone fractures in patients undergoing delayed reduction.

\section{MATERIALS AND METHODS}

Patients

Between March 2005 and August 2006, 15 patients who missed the optimal reduction time were selected for endoscopic open reduction. Their ages ranged from 14 to 48 years. For all patients, lateral and Water's view X-rays and computed tomography (CT) were obtained preoperatively to evaluate the type and extent of the fracture. CT was performed in the axial and coronal positions with slice intervals and slice thicknesses of $2 \mathrm{~mm}$. The patients' nasal deformities were all photographed preoperatively.

\section{Surgery}

Under general anesthesia, the patients were placed in the supine position. After local infiltration of the caudal border of the lateral cartilage, bilateral intercartilaginous incisions were made between the alar and upper lateral cartilages. Converse scissors were used to dissect the soft tissues from the lateral 


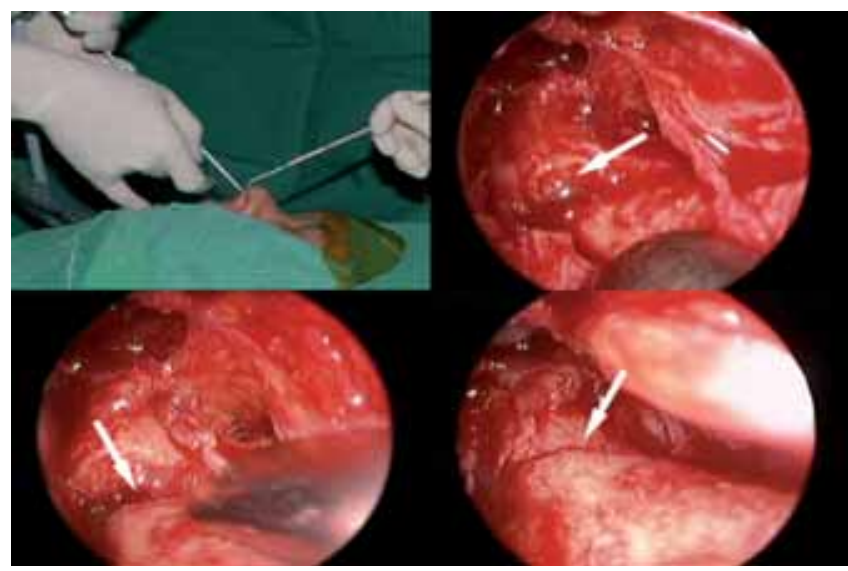

Figure 1. Intraoperative findings: (A) The tip of the $30^{\circ}$ endoscope is placed under elevation of the periosteal flap by L-shape retractor and the Boice elevator move in nasal fracture site . Endoscopic photographs showing (B) fibrotic change and callus formation at the fracture site(white arrow), (C) a depressed bone fragment, and (D) good alignment of the bone fragment after reduction(white arrows).

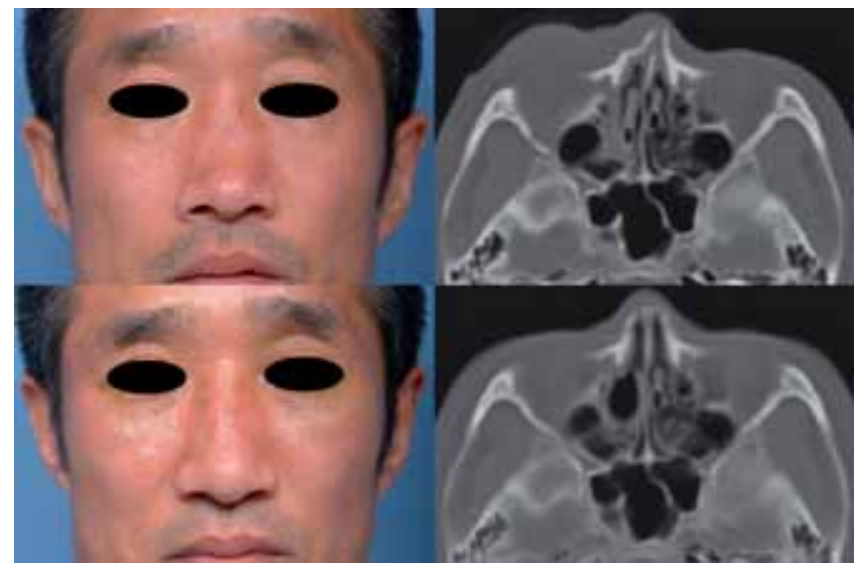

Figure 2. A 56-year-old man with a severe comminuted fracture. He had a reverse C-shaped dorsal deviation. The CT showed multiple fragments and dislocation of the nasal pyramid(Above). Three months after reduction, correction of the deviated nose with a very good external appearance (below).

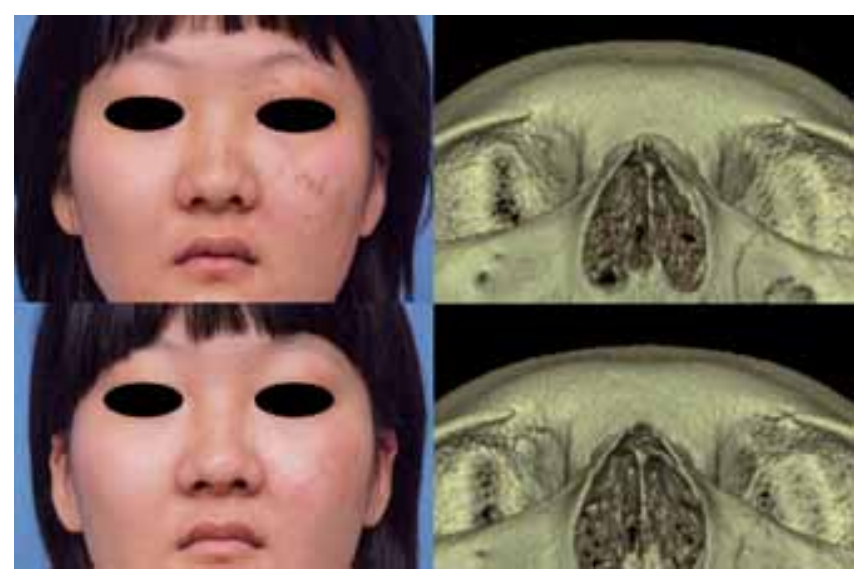

Figure 3. A 15-year-old woman with a laterally depressed fracture. She had a narrow midvault, and dorsal aesthetic lines that was disrupted in the keystone area. The 3D-CT showed depressed bony fragment of the nasal pyramid (above). Three months after reduction, the external shape of the nose is good with no depression and the 3D-CT shows good alignment of the nasal pyramid (below). cartilage to the caudal border of the fractured nasal bone. Bleeding was controlled with Bosmin ${ }^{\circledR}(1 \mathrm{mg} / \mathrm{mL}$ epinephrine solution; Je Il Pharm, Seoul, Korea) pledget. A 4-mm, 30 endoscope was inserted through the incision (Figure 1A), and a Freer's periosteal elevator was used to elevate the periosteum under endoscopic visualization (Figure 1B). The fracture lines and bony fragments of the nasal bone were easily detected with magnification on the monitor (Figure 1C). The displaced bone segments were lifted upward and outward with a Boies elevator, and accurate reduction was confirmed on the monitor (Figure 1D).

Intranasal packing was inserted to support the bone fragment, which was left in place for 3 days, and a Denver splint was applied to protect the nose for 2 weeks.

\section{Post-operative evaluation}

Three months postoperatively, we evaluated the nasal symmetry using external photographs and CT when possible.

\section{RESULTS}

We treated 10 males and 5 females with delayed reduction. The median time from injury to surgery was 27 days (range 2130 days). This interval was mainly determined by the patient's late presentation resulting from ignorance of the appropriate timing of reduction. The most common cause of injury was assault in seven cases, followed by traffic accidents in four cases. The main clinical symptoms were mild nasal deviation and pain. In all cases, the displacement of the nasal bone was evaluated by facial CT. During repositioning, the alignment of the nasal bone could be precisely assessed with the use of magnification on the monitor. In all of the patients, we could confirm the boundary of fracture site after elevating the fibrous connective tissue and periosteum. The mean operating time was 35 minutes, and the endoscopic procedure took $20 \mathrm{~min}$ utes. Three months postoperatively, the patients were evaluated using CT or photographs. No secondary deformities, such as saddling and hump nose, were present. Most of patients had a good nasal dorsum contour that was correct anatomically, and all of the patients were satisfied with the shapes of their noses (Figures 2 and 3). The mean patient satisfaction score was 9.4, where 1 indicated extreme dissatisfaction and 10 great satisfaction. No postoperative complications occurred during the mean postoperative follow-up period of 1 year (Table 1).

\section{DISCUSSION}

The projection of the nose on the face makes it highly vulnerable ${ }^{(1,2)}$ and some authors have reported that $39 \%$ of facial fractures involve the nose ${ }^{(2)}$. Since the nose is at the center of the face, any deformity after an injury is most noticeable and greatly affects the person's appearance. Therefore, proper treatment is needed to achieve good aesthetic results.

Several methods exist for treating nasal bone fractures, including closed reduction, open reduction, and rhinoplasty. Nasal 
Table 1. Data on the 15 patients.

\begin{tabular}{|c|c|c|c|c|c|c|}
\hline Patient No. & Sex/age & Cause & $\begin{array}{l}\text { Time from trauma } \\
\text { to surgery (days) }\end{array}$ & $\begin{array}{l}\text { Additional } \\
\text { procedure }\end{array}$ & $\begin{array}{c}\text { Patient } \\
\text { Satisfaction } \\
\text { score } \\
\end{array}$ & $\begin{array}{l}\text { Follow-up } \\
\text { (months) }\end{array}$ \\
\hline 1 & $\mathrm{M} / 14$ & Assault & 30 & & 9 & 11 \\
\hline 2 & $\mathrm{M} / 34$ & Rolling down & 26 & Septoplasty & 10 & 12 \\
\hline 3 & $\mathrm{~F} / 29$ & Assault & 21 & & 9 & 16 \\
\hline 4 & $\mathrm{M} / 17$ & Traffic accident & 28 & & 9 & 9 \\
\hline 5 & $\mathrm{~F} / 16$ & Fall down & 27 & & 10 & 6 \\
\hline 6 & $\mathrm{M} / 46$ & Assault & 27 & Septoplasty & 9 & 18 \\
\hline 7 & $\mathrm{M} / 16$ & Traffic accident & 29 & & 9 & 11 \\
\hline 8 & $\mathrm{~F} / 18$ & Assault & 30 & Septal spur removal & 10 & 13 \\
\hline 9 & $\mathrm{M} / 27$ & Traffic accident & 22 & & 10 & 10 \\
\hline 10 & $\mathrm{~F} / 35$ & Assault & 29 & & 10 & 15 \\
\hline 11 & $\mathrm{M} / 35$ & Traffic accident & 27 & & 9 & 12 \\
\hline 12 & $\mathrm{M} / 30$ & Fall down & 26 & & 10 & 11 \\
\hline 13 & $\mathrm{M} / 24$ & Assault & 29 & Spetoplasty & 8 & 10 \\
\hline 14 & $\mathrm{M} / 48$ & Fall down & 25 & & 9 & 12 \\
\hline 15 & $\mathrm{~F} / 42$ & Assult & 30 & Septal spur removal & 10 & 10 \\
\hline
\end{tabular}

fractures are managed in different ways, depending on the surgeon's experience, hospital protocols, and practical reasons. The standard treatment since antiquity has been closed reduction because it is simple and easy ${ }^{(3-5)}$, but the nasal bone is covered by a soft tissue envelope that makes palpation difficult (6). Consequently, in many situations, closed reduction alone does not give optimal results. Some authors advocate early rhinoplasty ${ }^{(7)}$. But in early rhinoplasty, the surgeon must be cautious regarding an uncontrolled fracture at the time of osteotomy, and bone resorption. In particular, unexpected bone resorption can result in inequality of the lateral nasal bone segment and shortening of the nasal bone, which can result in nasal deformity.

Conventionally, reduction is performed within 10 days, and rhinoplasty after 6 months ${ }^{(1,3)}$. A delay, however, often occurs before the patient seeks treatment. If patients miss the optimal time for reduction, they may have to wait months for the fracture site to stabilize. In these patients, the bone healing process and fibrous connective tissue in the fracture site will affect the anatomic reduction, necessitating complete removal of the fibrous tissue for an accurate reduction. In a closed reduction, the intraoperative assessment of bone fragment repositioning is based on palpation and the facial contour only. Therefore, an additional technique for intraoperative examination may be required to correct the bone fragments (ie. endoscopic technique).

In 1994, Mitz introduced endoscopic visualization in rhinoplasty ${ }^{(8)}$, and in 2001, Kim and Kim reported that the endoscopic approach can solve the problems of blind procedures in conventional corrective rhinoplasty ${ }^{(9)}$. Some authors emphasize that hemostasis with epinephrine gauze and irrigation are important for adequate visualization. Others obtain visualization using an intraoral approach. We obtained good visualiza- tion with Bosmin ${ }^{\circledR}$ gauze via an intercartilaginous incision. Some authors report that the endoscopically assisted transnasal approach is more time-consuming because of the nasal restructuring maneuvers and extra time required for inserting and removing the endoscopy ${ }^{(6,9)}$. In our experience, the mean operating time was 35 minutes in 15 patients who underwent endoscopically assisted open reduction compared to $15 \mathrm{~min}$ utes in eight patients who underwent closed reduction. The endoscopic procedure takes only 20 minutes. Endoscopy enables precise diagnosis of the fracture site, confirmation of any fibrotic change at the fracture site, accurate reduction, and immediate evaluation of the fracture reduction because the fracture lines and bony segments are easily detected with magnification on the monitor. The other advantage of our reduction method is the ability to reduce the small bone fragments.

\section{CONCLUSION}

Although the additional operating time is a disadvantage, endoscopically assisted reduction is a useful, reliable method for the treatment of nasal fractures because it achieves accurate reduction. Endoscopically assisted reduction provides an alternative for the treatment of patients who miss the optimal time for reduction.

\section{ACKNOWLEDGEMENT}

This work was supported by a grant (code \#200810 FTH010103002) from the BioGreen 21 Program, Rural Developement administration, Republic of Korea.

\section{REFERENCES}

1. Tremolet de Villers Y. Nasal fractures. J Trauma. 1979; 15: 319327.

2. Fremandes SV. Nasal fractures: the taming of the shrewd. Laryngoscope. 2004; 114: 587-592.

3. Ridder GJ, Boedeker CC, Fradis M, et al. Technique and timing for closed reduction of isolated nasal fractures: a retrospective study. Ear Nose Throat J. 2002; 81: 49-54. 
4. Mondin V, Rinaldo A, Ferlito A. Management of nasal bone fractures. Am J Otolaryngol. 2005; 26: 181-185.

5. Staffel JG. Optimizing treatment of nasal fractures. Laryngoscope. 2002; 112: 1709-1719.

6. Park DH, Kim TM, Han DG, et al. Endoscopic-assisted correction of the deviated nose. Aesthetic Plast Surg. 1998; 22: 190-195.

7. Gregory JR. Management of nasal fractures. In: Robert AW, Robert BX, editors. Current Issues in Head and Neck Trauma. The Otolaryngologic Clinics of North America; Philadelphia, WB Saunders; 1991: 195-213.

8. Mitz V. Endoscopic control during rhinoplasty. Aesthetic Plast Surg. 1994; 18: 153-156.

9. Kim JT, Kim SK. Endoscopically assisted, intraorally approached corrective rhinoplasty. Plast Reconstr Surg. 2001; 108: 199-205.
Chan Hum Park, MD

Department of Otorhinolaryngology-Head and Neck Surgery

Chuncheon Sacred Heart Hospital

School of Medicine, Hallym University \#153, Kyo-Dong, Chuncheon

Kangwon, Korea

Tel: +82-33-240-5181

Fax: +82-33-241-2909

E-mail: hlpch@paran.com

\section{ANNOUNCEMENT}

$23^{\text {rd }}$ Congress of the European Rhinologic Society

$29^{\text {th }}$ International Symposium of Infection \& Allergy of the Nose

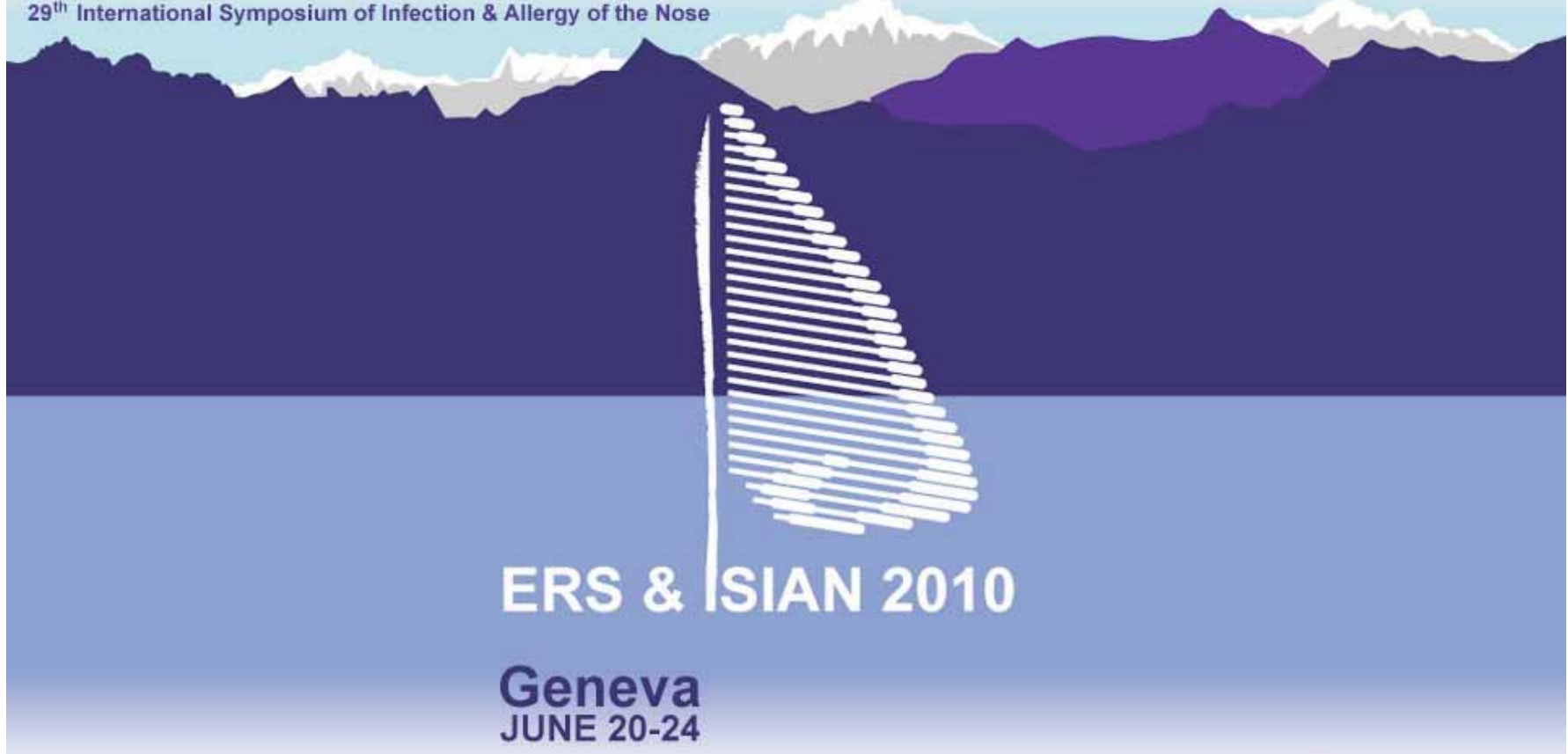

JUNE 20-24 